\title{
Traços psicológicos dos pacientes submetidos a angioplastia transluminal coronária
}

\author{
Lourdes Helena de CAMPOS*, Gláucia Faé NASCIMENTO*
}

RBCCV 44205-125

CAMPOS, L. H. \& NASCIMENTO, G. F. - Traços psicológicos dos pacientes submetidos a angioplastia transluminal coronária. Rev. Bras. Cir. Cardiovasc, 5(3): 195-199, 1990.

RESUMO: Este trabalho teve por objetivo a identificação de traços psicológicos e características emocionais comuns aos pacientes que foram, pela primeira vez, submetidos a angioplastia transluminal coronária (ATC), no Instituto Dante Pazzanese de Cardiologia. Foram analisados 84 pacientes, de ambros os sexos, com idade média de 55 anos. Foram realizadas, pela equipe de psicólogos, duas entrevistas semidirigidas, durante cada uma das quais foram aplicados o Inventário de Ansiedade Traço - Estado (IDATE) e a Escala de Hamilton. Essas entrevistas foram realizadas imediatamente anterior à ATC e um dia após o procedimento, na alta dos pacientes. Os resultados permitiram-nos concluir que a grande maioria apresentou padrāo comportamental tipo A, próprio do coronariano, alto estresse constitucional e ambiental e ansiedade - traço acima da média. Obtiveram alta porcentagem, também, as variáveis tensão, depressāo, grau de competitividade e labilidade de humor.

DESCRITORES: miocardio, revascularizaçāo, análise psicológica de pacientes; angioplastia transluminal coronária, análise psicológica de pacientes; análise psicológica, pacientes coronarianos.

\section{INTRODUÇĀO}

As doenças coronarianas apresentam uma incidência crescente entre nós. É característica da vida moderna, da longevidade e do ritmo crescente de solicitação individual, frente a uma sociedade cada vez mais competitiva.

No campo da psicologia, várias pesquisas têm sido direcionadas ao estudo do coronariano a ser revascularizado cirurgicamente. Os resultados têm demonstrado importantes aspectos psíquicos, que se interrelacionam aos aspectos orgânicos, ajudando, de forma comprovada, a prática médica quanto ao maior entendimento de processos e funcionamentos mentais e emocionais, contribuindo para a compreensāo de um indivíduo total. Traços emocionais desses pacientes foram, inicialmente, estudados por FRIEDMAN \& ROSENMAN ${ }^{4}$, que identifi- caram vínculos estreitos entre comportamentos específicos e transtornos cardiovasculares, características de indivíduos de padrāo comportamental tipo A. Traços específicos, como repressão, ansiedade e sensibilidade, também foram amplamente estudados em coronarianos. Demonstraram que procedimentos médicos estressantes encontram diferentes padrōes de respostas, de acordo com a características específica do traço estudado nesses pacientes. O tipo de informação recebida é também um aspecto comprovadamente importante. Traços emocionais específicos foram relacionados ao nível de informação recebida por coronarianos, mostrando diferenças acentuadas de respostas em relação à adaptação ao tratamento médico. Estudos realizados com pacientes com infarto do miocárdio demonstram diferentes relaçōes entre niveis de informação médica e indivíduos reprimidos, levando-os

Trabalho realizado no Instituto Dante Pazzanese de Cardiologia. São Paulo. SP, Brasil.

Recebido, para publicaçâo em $1^{\circ}$ de dezembro de 1990.

* Do Instituto Dante Pazzanese de Cardiologia.

Endereço para separatas: Lourdes Helena de Campos. Av. Dr. Dante Pazzanese, 500. 04012 São Paulo, SP. Brasil. 
CAMPOS, L. H. \& NASCIMENTO, G. F. - Traços psicológicos dos pacientes submetidos a angioplastia transluminal coronária. Rev. Bras. Cir. Cardiovasc, 5(3): 195-199, 1990.

a uma deterioraçāo nos resultados médicos, psicomédicos e psicossociais $1-3,6,7,9,10,13$.

SHAW et alli ${ }^{8}$ pesquisaram a relaçāo entre estresses e níveis de ansiedade, caracterizando ainda mais os traços psicológicos de indivíduos coronarianos.

Sabe-se que, atualmente, dos pacientes com indicação para cirurgia de ponte de safena, cerca de $50 \%$ têm sido tratados pela dilataçāo coronária transluminal.

Segundo os dados do Instituto Nacional do Coraçāo, Sangue e Pulmāo (USA) ${ }^{6}$ de 3000 pacientes submetidos a angioplastia transluminal coronária, 20 a $30 \%$ apresentaram taxas de reestenose durante os seis primeiros meses após a intervençāo em que todos os aspectos físicos foram controlados. Estes dados mostram a necessidade de um estudo específico desses pacientes a nível psicológico, indicando a importância do conhecimento dos vários aspectos emocionais, principalmente na relaçāo médico/paciente.

Embora os trabalhos em psicologia realizados com coronarianos sejam em grande número, existem poucas informaçōes a respeito da dinâmica emocial de pacientes submetidos ao procedimento de angioplastia. O levantamento bibliográfico realizado indicou somente uma publicaçāo, em 1986, relacionada a pacientes que se submeteram a angioplastia transluminal coronária. SHAW et alli ${ }^{8}$ relacionam, nesse trabalho, variáveis psicológicas, tais como traços repressivos, ansiedade e nível de informaçāo sobre o procedimento, com má evoluçāo tardia (reestenose).

No presente estudo, foram abordados traços e características psicológicas apresentadas por pacientes do Instituto Dante Pazzanese de Cardiologia, submetidos à ATC. Teve como objetivo principal fornecer subsídios para uma atuaçāo psicológica institucional, a nível assistencial/preventivo no Setor de Angioplastia, e servir de base para outros estudos relacionados à área de Psicologia aplicados em pacientes do referido setor.

\section{CASUÍSTICA E MÉTODOS}

A amostra foi constituída por 84 pacientes de ambos os sexos, matriculados no Instituto Dante Pazzanese de Cardiologia, submetidos pela primeira vez à angioplastia transluminal coronária (ATC) e obtiveram sucesso no procedimento.

Esses pacientes foram submetidos a exames clínicos e laboratoriais, sendo encaminhados pelos cardiologistas do setor aos psicólogos. Foram sub- metidos a uma primeira entrevista pré-ATC, com aplicaçăo da Escala de Avaliaçăo de Ansiedade de HAMILTON ${ }^{5}$ e parte I do IDATE (Inventário de Ansiedade Traço-Estado) ${ }^{11}$. A segunda entrevista foi realizada após confirmaçāo do sucesso da ATC e por ocasiāo da alta médica, com a reaplicação da parte I do IDATE e aplicaçāo da parte II desse Inventário.

Foi utilizada a técnica de entrevistas semidirigidas, solicitando-se aos pacientes que falassem sobre suas dificuldades e preocupações, principalmente relacionadas ao procedimento da ATC. Áreas exploradas, também, foram as que corresponderam a fatores de possível interferência no estado emocional dos pacientes, como a percepção da enfermidade anterior (pré ATC) e posterior (pós ATC), procurando-se determinar o momento de crise psicológica correspondente ao início dos sintomas, nível de informaçāo sobre a angioplastia, mudanças com relaçāo às áreas de saúde, expectativas e qualidade de vida.

O IDATE (Inventário de Ansiedade Traço-Estado) de Spilberger, Gorsuch e lushene é composto por duas escalas distintas de auto-relatório. Foram elaboradas para medir quantitativamente dois conceitos distintos de ansiedade: estado de ansiedade $(A-E)$ e traço de ansiedade (A-T).

A Escala de Avaliação de Ansiedade de Max Hamilton tem por objetivo a avaliaçāo qualitativa da ansiedade e do estado ansioso e de dois grupos de manifestaçōes: a) psíquicas (tensão, medos, insônia, dificuldades intelectuais, humor); b) somáticas (sintomas musculares, sensoriais, cardiovasculares, respiratórios, gastrintestinais, geniturinários e do sitema nervoso autônomo)

Durante a aplicaçāo dessa escala, sāo observados comportamentos apresentados pelos pacientes. As variáveis sāo avaliadas e classificadas de acordo com as respostas nos níveis: ausente, leve, moderado, grave, ou incapacitado.

A avaliaçāo dos níveis dos estresses (constitucional, ambiental e particular) foi avaliada na entrevista, a partir de respostas do paciente ao roteiro de avaliação de estresse. No estresse constitucional foram pesquisados comportamentos típicos do padrāo comportamental $A$, desde a marcada tendência para competiçāo até a aceleraçāo do ritmo de tarefas mentais e físicas. No estresse ambiental foram analisadas as influências exercidas pelo meio ambiente e agentes estressores relacionados à atmosfera social que agem negativamente na parte emocional do paciente. Conflitos internos do paciente e conseqüente instabilidade emocional, relacionados à vida familiar e ocupacional foram estressores particulares também pesquisados. 
CAMPOS, L. H. \& NASCIMENTO, G. F. - Traços psicológicos dos pacientes submetidos a angioplastia transluminal coronária. Rev. Bras. Cir. Cardiovasc, 5(3): 195-199, 1990.

Todos os dados coletados foram transferidos para fichas individuais e computados posteriormente em ficha única.

\section{RESULTADOS}

$\mathrm{Na}$ Tabela 1 encontramos dados de identificação dos pacientes.

\section{TABELA 1}

DADOS DE IDENTIFICAÇĀO DE PACIENTES DO SETOR DE ANGIOPLASTIA DO INSTITUTO DANTE PAZZANESE DE CARDIOLOGIA, ENTREVISTADOS PELA SEÇĀO DE PSICOLOGIA

\begin{tabular}{lrlr}
\hline $\begin{array}{l}\text { Sexo masculino: } \\
\text { Idade Média: }\end{array}$ & $\mathbf{7 9 , 8 \%}$ & ESTADO CIVIL & \\
& 55 a & $\begin{array}{l}\text { Casados } \\
\text { Separados }\end{array}$ & $76,2 \%$ \\
ESCOLARIDADE & & $\begin{array}{l}\text { Viúvos } \\
\text { Sem escolaridade }\end{array}$ & $10,8 \%$ \\
Primário & $8,4 \%$ & Solteiros & $3,6 \%$ \\
Ginasial & $37,0 \%$ & ATIVIDADE LABORATIVA \\
Colegial & $13,1 \%$ & ATIV & $68,9 \%$ \\
Superior & $16,7 \%$ & Ativos & $32,2 \%$ \\
\hline
\end{tabular}

A Tabela 2 mostra que $64,3 \%$ dos pacientes alcançaram escores acima da média em A-T, que é uma característica comportamental de indivíduos coronarianos de padrāo Tipo A e que pós ATC, $25 \%$ desses pacientes mantiveram níveis acima da média em A-E.

\section{TABELA 2}

NIVEIS DE ANSIEDADE TRAÇO-ESTADO ACIMA DA MÉDIA EM PACIENTES DO SETOR DE ANGIOPLASTIA DO INSTITUTO DANTE PAZZANESE DE CARDIOLOGIA

Ansiedade-traço acima da média:

$64,3 \%$

Ansiedade-estado acima da média:

$\begin{array}{ll}\text { Pré-ATC } & 48,8 \% \\ \text { Pós-ATC } & 25,0 \%\end{array}$

Notou-se, também, que mais da metade dos pacientes sofrem constitucional $(62,9 \%)$ e ambiental $(83,4)$, como mostra a Tabela 3 . Eventos como aceitação de uma carga excessiva de trabalho, responsabilidade profissional, desmotivação e insatisfação com a atividade exercida, foram dados significativos, como também o grau de competitividade, instabilidade profissional e falta de segurança pessoal.
TABELA 3

NIVEIS DE ESTRESSES CONSTITUCIONAL, AMBIENTAL E PARTICULARES EM PACIENTES DO SETOR DE ANGIOPLASTIA DO INSTITUTO DANTE PAZZANESE DE CARDIOLOGIA

ESTRESSE

$\begin{array}{ll}\text { Constitucional } & 62,9 \% \\ \text { Ambiental } & 83,4 \% \\ \text { Particulares } & 42,9 \%\end{array}$

Foram correlacionados níveis de ansiedade-traço acima da média (A-T aumentado) com os níveis de estresses (Tabela 4). A maior relação obtida foi entre estresse ambiental e A-T aumentado, indicando que eventos negativos do meio afetam mais pacientes que, constitucionalmente, respondem com alta ansiedade a estímulos externos e internos.

TABELA \&

CORRELAÇĀO ENTRE ESTRESSES E ANSIEDADETRAÇO ACIMA DA MÉDIA EM PACIENTES DO SETOR DE ANGIOPLASTIA DO INSTITUTO DANTE PAZZANESE DE CARDIOLOGIA

ESTRESSES + ANSIEDADE-TRAÇO ACIMA DA MÉDIA

\begin{tabular}{|c|c|c|}
\hline & AT & aumentado: $63,0 \%$ \\
\hline & AT & do: 87,0 \\
\hline & AT & aumenta \\
\hline
\end{tabular}

EC - estresse constitucional

EA - estresse ambiental

EP - estresse particular

Outros dados foram correlacionados aos tipos de estresses e os resultados mostram que o estresse ambiental afeta pacientes de diferentes atividades laborativas, estado civil, sexo e faixa etária, de acordo com as Tabelas $5,6,7$ e 8 .

As procentagens encontradas nas variáveis tensão, depressão, pânico e labilidade de humor, foram pesquisadas através da Escala de Hamilton (Tabela 9). Quanto à tensão, os pacientes relata-

TABELA 5

CORRELAÇĀO ENTRE ESTRESSES E ESTADO CIVIL EM PACIENTES DO SETOR DE ANGIOPLASTIA DO INSTITUTO DANTE PAZZANESE DE CARDIOLOGIA

CORRELAÇĀO ENTRE ESTRESSES E ESTADO CIVIL

Estado Civil Estresse Constituticional Estresse Ambiental

$\begin{array}{lrr}\text { casados } & 53,2 \% & 81,3 \% \\ \text { viúvos } & 33,4 \% & 88,9 \% \\ \text { solt./separados } & 100,0 \% & 90,9 \%\end{array}$


CAMPOS, L. H. \& NASCIMENTO, G. F. - Traços psicológicos dos pacientes submetidos a angioplastia transluminal coronária. Rev. Bras. Cir. Cardiovasc, 5(3): 195-199, 1990.

TABELA 6

CORRELAÇÄO ENTRE ESTRESSES CONSTITUCIONAL (EC) E AMBIENTAL (EA) E TRABALHO EM PACIENTES DO SETOR DE ANGIOPLASTIA DO INSTITUTO DANTE PAZZANESE DE CARDIOLOGIA

CORRELAÇĀO ENTRE ESTRESSES E TRABALHO

Estresses nos que nāo trabalham

$\begin{array}{ll}\text { EC } & 29,7 \% \\ \text { EA } & 88,9 \%\end{array}$

Estresses nos que trabalham

EC $\quad 70,2 \%$

EA $\quad 80,7 \%$

TABELA 7

CORRELAÇĀO ENTRE ESTRESSES CONSTITUCIONAL (EC) E AMBIENTAL (EA) E SEXO EM PACIENTES DO SETOR DE ANGIOPLASTIA DO INSTITUTO DANTE PAZANESE DE CARDIOLOGIA

CORRELAÇĀO ENTRE ESTRESSE E SEXO

$\begin{array}{lll}\text { SEXO } & \text { EC } & \text { EA } \\ \text { Masc. } & 64,3 \% & 83,9 \% \\ \text { Fem. } & 7,1 \% & 78,5 \%\end{array}$

TABELA 8

CORRELAÇĀO ENTRE ESTRESSES CONSTITUCIONAL (EC) E AMBIENTAL (EA) E FAIXA ETÁRIA EM PACIENTES DO SETOR DE ANGIOPLASTIA DO INSTITUTO DANTE PAZZANESE DE CARDIOLOGIA

CORRELAÇĀO ENTRE ESTRESSES E FAIXA ETÁRIA

$\begin{array}{llc}\text { IDADE } & \text { EC } & \text { EA } \\ 30-50 & 90,9 \% & 95,5 \% \\ 51-70 & 44,5 \% & 77,8 \% \\ 71- & 50,0 \% & 87,5 \%\end{array}$

TABELA 9

PORCENTAGENS ENCONTRADAS NAS VARIÁVEIS PESQUISADAS EM PACIENTES DO SETOR DE ANGIOPLASTIA DO INSTITUTO DANTE PAZZANESE DE CARDIOLOGIA

\begin{tabular}{lc}
\hline VARIÁVEIS & PORCENTAGENS \\
Tensão & $62,0 \%$ \\
Depressão & $46,5 \%$ \\
Pânico & $27,4 \%$ \\
Competitividade & $44,1 \%$ \\
Labilidade de humor & $50,0 \%$ \\
\hline
\end{tabular}

ram, sobre o estado e, principalmente, sobre o humor ansioso, inquietudes, irritabilidade e apreensōes quanto ao presente e ao futuro, de forma constante. Do mesmo modo, relatos sobre a nāo percepçāo do estado de tensāo e incapacidade de obter relaxamento foram quase unânimes. Quanto aos estados depressivos, os dados mais significativos referiramse à perda de interesse, variaçāo de humor $e$ indiferença às atividades de rotina.

\section{COMENTÁRIOS}

A análise dos dados referentes aos estresses revelou que os eventos ambientais obtiveram alta incidência entre os pacientes, demonstrando que as influências sofridas pelo meio em que vivem agem de forma negativa no organismo. Estressores, como instabilidade profissional e/ou financeira, alto grau de competiçāo social, dificuldades no trânsito e falta de segurança, foram constantes e signinicativos durante as entrevistas.

Em relação à competitividade, os pacientes relataram que, mesmo nāo trabalhando em atividade onde o nível de competiçāo era alto, sentiam-se impelidos a realizar mais tarefas e a trabalhar mais horas do que eram necessárias, mesmo sem conseguir obter satisfaçäo. O aumento do rítimo de tarefas mentais, inclusive em período de férias, foram relatos significativos.

Dados qualitativos e quantitativos mostram que o nível de ansiedade pré-ATC atingem escores altos em quase metade dos pacientes estudados. A comparaçāo entre os níveis de ansiedade pré-ATC e pós-ATC indicam que o procedimento de angioplastia gera forte tensăo e age como importante evento estressor. Para a maioria desse grupo de coronarianos e sua conhecida dinâmica psicológica, seria indicada a Psicoterapia Breve com foco dererminado acerca da sintomatologia e o uso mental que o paciente faz deste. O objetivo é o de promover mudanças e obter recursos internos, a fim de compreender o significado da doença e melhorar as condiçōes mentais de lidar com esta, na busca de um maior equilíbrio emocional.

Comportamentos repetidos frente a estímulos agressivos externos, mantêm tendência à uniformidade do tipo de reação e expressão de emoçōes do paciente em relação ao meio em que vive. Propōe-se um trabalho psicológico posterior à ATC, a nível profilático, promovendo mudanças nessa forma de recepçāo de eventos ambientais agressores, para que as descargas emocionais encontrem meios de expressāo mais adequados. Possibilitaria, ainda, ao paciente maior tempo para esclarecer seus próprios conflitos, a percepçāo do seu 
CAMPOS, L. H. \& NASCIMENTO, G. F. - Traços psicológicos dos pacientes submetidos a angioplastia transluminal coronária. Rev. Bras. Cir. Cardiovasc, 5(3): 195-199, 1990.

estado de tensão constante e a incapacidade para o relaxamento, adaptando melhor esse paciente à realidade.

Assim sendo, ao paciente dever-se-ia oferecer apoio psicológico em forma de psicoterapia breve durante o período de preparação que precede a ATC, possibilitando chance de sucesso maior, sem alteraçăo da rotina existente para esses casos, ou maior demanda de tempo.

\section{RBCCV 44205-125}

CAMPOS, L. H. \& NASCIMENTO, G. F. - Psychological profile of patients that underwent coronary angioplasty. Rev. Bras. Cir. Cardiovasc., 5(3): 195-199, 1990.

ABSTRACT: This paper presents psychological and emotional characteristics that were found common to patients that underwent a first coronary angioplasty at Instituto Dante Pazzanese de Cardiologia. We studied 84 patients of both sexes, with mean age of 55 years. Two semi-directed interviews were conducted by the psychology team, and during each of them the State-Trait - Anxiety Inventory STAI and the Hamilton Scale were applied. Such interviews were conducted immediately before PTCA and repeated one day after it, at patient's discharge. We concluded that most patients had a type A personality, typical of patients with coronary artey disease, high constitutional stress and anxiety-trace higher than the average population. There was also a high incidence of tension, depression, competitivity and humor lability.

DESCRIPTORS: myocardial revascularization, psychological profile of patients; angioplasty, coronary, psychological profile of patients; psychological profile, coronarian patients.

\section{REFERÊNCIAS BIBLIOGRÁFICAS}

1 ASENDORPF, J. B. \& SCHERER, K. R. - The discrepant repressor differentiation between low anxiety, high anxiety, and repression of anxiety by autonomicfacial-verbal patterns of behavior. J. Pers. Soc. Psychol., 45: 1334-1346. 1983.

CROMWELL, R. L.; BUTTERFIELD, D. C.; BRAYFIELD, F. M.; CURRY, J. J. - Acute myocardial infarction: reaction and recovery. St. Louis, Mosby, 1977.

3 CROWNE, D. P. \& MARLOWE, D. - A new scale of social desiderability independent of psychopathology. J. Consult. Psychol., 24: 349-354, 1960.

4 FRIEDMAN, M. \& ROSENMAN, R. H. - Stress, comportamento tipo $A$ e modelo bioquímico na aterosclerose coronária. AC Cardiologia, 5: 25-33, 1983.

5 HAMILTON M. - The assessment of anxiety states by rating. Br. J. Med. Psychol., 32: 50-55, 1959.

6 The National, Heart, Lung and Blood Institute's PTCA Registry. N. Engl. J. Med., 318: 265-270, 1988.

7 SHAW, R. E.; COHEN, F.; DOYLE, B.; PALESKY, J. The impact of denial and repressive style on information gain and rehabilitation outcomes in myocardial infarction patients. Psychosom. Med., 47: 262-273, 1985.
8 SHAW, R. E.; COHEN, F.; FISHMAN-ROSSEN, J.; MURPHY, M. C.; STERTZER, S. H.; CLARK, D. A.; MYLER, R. K. - Psychologic predictors of psychosocial and medical outcomes in patients undergoing coronary angioplasty. Psychosom. Med., 48: 582-597, 1986.

9 SHIPLEY, R. H.; BUTT, J. E.; HORWITZ, B. - Preparation to reexperience a stressful medical examination: effect of repetitions videotape exposure and copying style. J. Consult. Clin. Psychol., 47: 485492, 1979.

10 SHIPLEY, R. H.; BUTT, J. E.; HORWITZ, B.; FARBY, J. E. - Preparation for a stressful medical procedure: effect of amount of stimulus preexposure and copying style. J. Consult. Clin. Psychol., 46: 499-507, 1978.

11 SPIELBERGER, C. D.; GORSUCH, R. L.; IUSHENE, R. E. - Inventário de ansiedade traço-estado - IDATE - Manual de psicologia aplicada, traduçāo de Angela Biaggio e Luís Natalício. Rio de Janeiro, 1979.

12 TAYLOR, J. A. - A personality scale of manifest anxiety. J. Abnorm. Soc. Psychol., 48: 285-290, 1953.

13 WEINBERGER, D. A.; SCHWARTZ, G. E.; DAVIDSON, J. R. - Low anxious, hight anxious, and depressive copying styles: psychometric patterns and behavioral physiological responses to stress. J. Abnorm. Psychol., 88: 369-380, 1979. 